\title{
Research on Desktop Delivery Device Based on Virtualization Technology
}

\author{
Jiang Jianjun ${ }^{+}$, Zhang Yingying, Lu Ping \\ Shanghai DianJi University, Shanghai, 201306, China
}

\begin{abstract}
This paper introduces a method of desktop delivery device based on virtualization technology[1].Desktop delivery device creates virtual desktop[2] and makes-desktop image through virtual desktop delivery management engines. When the thin client[3] requests, the virtual desktop is assigned to the thin client and recycled from the thin client; when the fat client requests,the desktop image is assigned to the fat client and recycled from the fat client; when the zero client requests,the client image is transferred to the zero client,and then works in the way of thin client or fat client.The method can support multiple clientsin one virtual desktop architecture.
\end{abstract}

Keywords: desktop virtualization; VDI; VOI; diskless boot technology

\section{Introduction}

In practical application,how desktop virtualization supports various clients is a problem to be solved.For thin client device,its computing capability is weak and it is suitable for VDI desktop virtualization applications.Because all virtual desktops are running on the server,the calculation is concentrated on the server,and it does not need to use a lot of computing capability of the client.For fat client device,because of its computing capability is stronger and it is more suitable for the virtualization applications of VOI mode[4].It can reduce the load on the virtualization server by taking advantage of the powerful computing capability of the fat client[5].Both thin and fat clients need software to support,and thus can boot the system to a point where they can connect to the virtualization server.This may be a complete operating system or a simplified operating system,but it provides an interface to the virtualization server.Such clients usually need more maintenance and management.In order to greatly reduce the management of the whole network operation,zero client is often used in the actual virtual desktop deployment scheme.Zero client[6] does not need any physical storage media (such as hard disk, flash memory, etc.).It can boot from the network through PXE[7] to grab an operating system image and run in memory.At present, the research and industrialization of desktop virtualization technology mainly focus on VDI,while the research on VOI is relatively less,and the problem of how to support various clients in a virtual desktop architecture has not been solved.

Therefore, in order to overcome the shortcomings of the existing technology,this paper provides a desktop delivery device implementation system based on virtualization technology.By integrating different virtual desktop implementation methods and diskless boot technology[8],it can provide virtual desktop support for various clients including thin client, fat client and zero client when they are deployed in LAN.

\section{Design of Desktop Delivery Device System}

The desktop delivery device includes a host operating system,virtualization software,a virtual desktop delivery management engine, a virtual server and a virtual desktop or desktop image or client image.The host operating system is installed in the desktop delivery device.The virtualization software and virtual desktop delivery management engine are installed in the host operating system,which turns the desktop delivery device into a virtualization platform. The desktop delivery device uses the virtual desktop delivery management engine to create a virtual machine,install a system for the virtual machine,make template based

Corresponding author. Tel.: + 18964587756; fax: +38223108.

E-mail address: jiangjj@sdju.edu.cn. 
on a virtual machine and create a virtual machine with the same configuration or produce a desktop image based on the template.The desktop delivery device allocates the virtual desktop to the thin client and retrieves it from the thin client when requested by the thin client,assigns the desktop image to the fat client and retrieves it from the fat client when requested by the fat client,and transfers the client image to the zero client for booting when requested by the zero client,then the zero client can work in the way of thin client or fat client.

Figure 1 shows a system architecture diagram for the desktop delivery device based on virtualization technology.

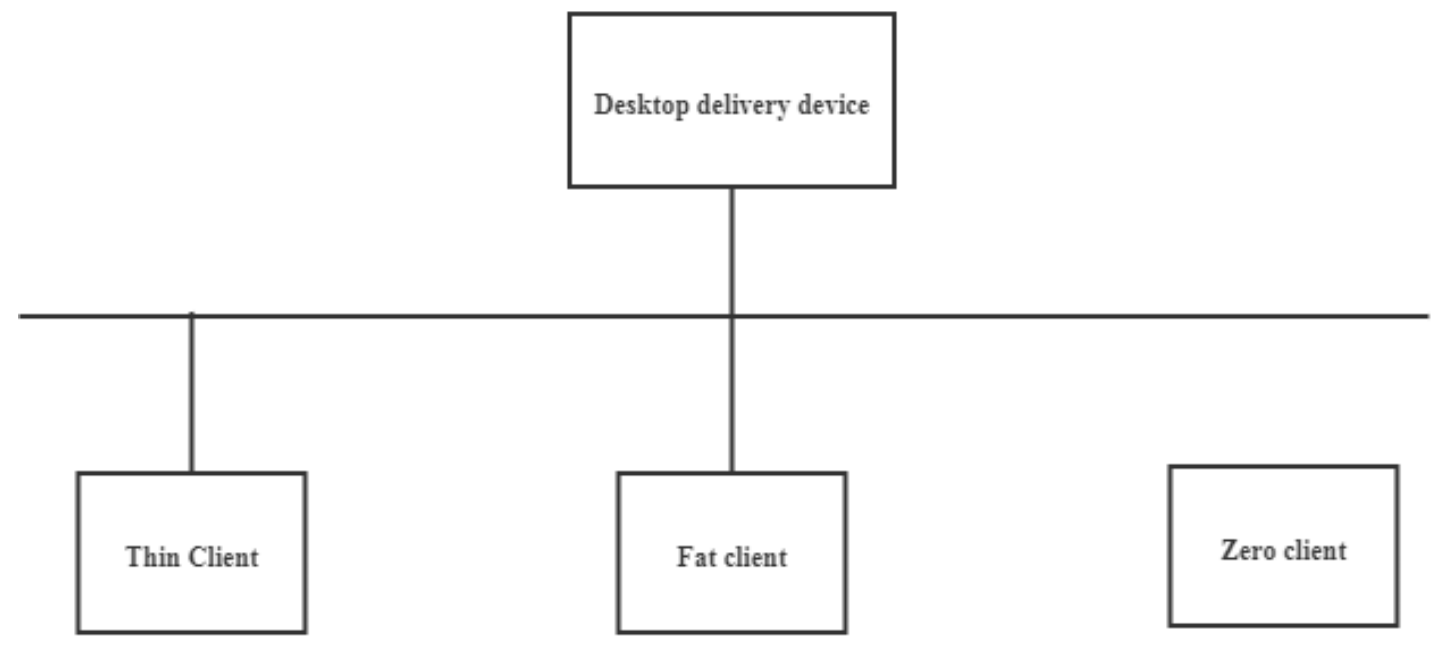

Fig. 1: System Architecture Diagram

The desktop delivery device connects thin client,fat client and zero client through network connection device.The desktop delivery device,thin client,fat client and zero client are deployed on the same LAN and communicate with each other based on network protocol.The virtual desktop runs as a process on the desktop delivery device and is connected remotely by the thin client via the transport protocol connection software.The desktop image is existing as a file on the desktop delivery device and is mounted to run in the local virtualization software of the fat client.Desktop delivery devices include virtual server for the diskless boot technology.The virtual server transfers the client image,which is placed in the desktop delivery device space to the zero client,so that the zero client can boot through this client image and connect to a virtual desktop on a desktop delivery device or mount the desktop image to run in local virtualization software.The desktop delivery device also includes a network bridge module so that the thin client can connect to the virtual desktop,the fat client can map the desktop image and the zero client can download the client image on it.

\section{A Desktop Delivery Device Implementation Approach}

A virtual machine to be created or a desktop image to be produced through the Virtual Desktop Delivery management engine,and the virtual machine can act as a virtual desktop or virtual server.According to the request of each client,the virtual machine or the desktop image is managed.The management operations of virtual desktops include creating virtual machines, installing systems for virtual machines,creating templates based on virtual machines,creating virtual machines of the same configuration based on templates and assigning virtual machines to thin clients and retrieving it from thin clients.The virtual desktop runs as a process on the desktop delivery device,connected remotely by thin clients via transport protocol connection software.The management operations for this desktop image include assigning the desktop image to the fat client and retrieving it from the fat client.The desktop image is existing as a file on the desktop delivery device and is mounted to run in the local virtualization software of the fat client.The virtual server is designed for zero client that uses diskless boot technology,and transfers the client image which is placed in the desktop delivery device space to the zero client, so that the zero client can boot through this client image 
and is connected to a virtual desktop on a desktop delivery device or mount the desktop image to run in local virtualization software.

Figure 2 shows the internal module structure diagram applied by the desktop delivery device.

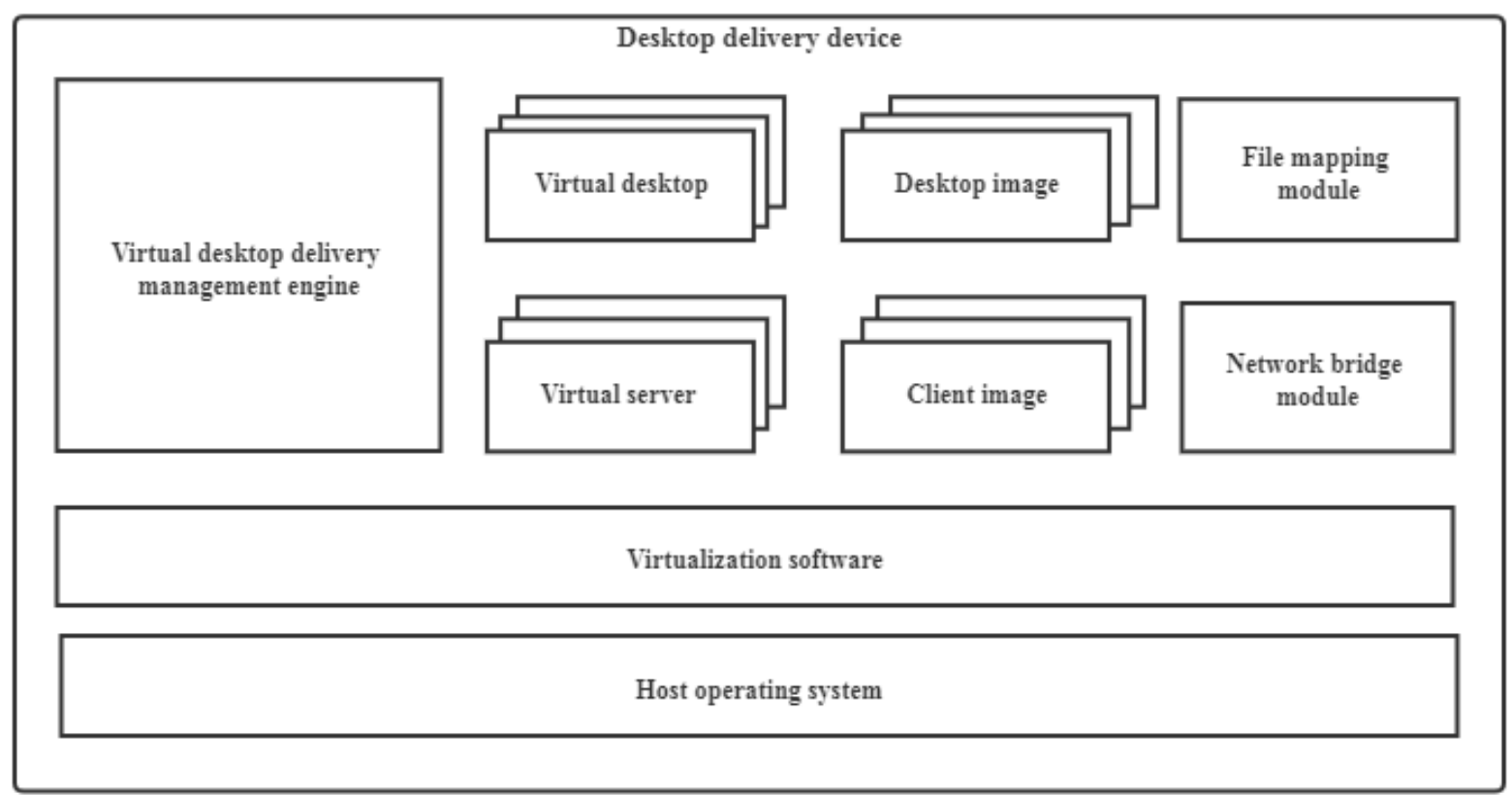

Fig. 2: Internal Module Structure Diagram

The specific implementation method of the desktop delivery device based on virtualization technology is as follows:

Create a virtual machine or produce a desktop image through the Virtual Desktop Delivery management engine.Different virtual machines can be used for different purposes, in which some are virtual desktops and some are virtual servers.The desktop image is actually the disk image file of the virtual machine,used with the file mapping module.

According to the request of each client,the virtual machine or desktop image is managed. The management operations of virtual desktops include creating virtual machines,installing systems for virtual machines,creating templates based on virtual machines,creating virtual machines of the same configuration based on templates and assigning virtual machines to thin clients and retrieving it from thin clients.The virtual desktop runs as a process on the desktop delivery device,connected remotely by thin clients via transport protocol connection software. This is the running mode of VDI (Virtual Desktop Infrastructure).The management operations for this desktop image include creating desktop image.According to the request of fat client, then assign the desktop image to the fat client and retrieve it from the fat client.Desktop image is mounted to and run on the local virtualization software of the fat client.This is the operation mode of VOI(virtual OS infrastructure).

As is shown in the figure 3,the network communication diagram includes the virtual machine,the desktop delivery device and the client.The virtual machines (virtual desktops and virtual servers) contain at least one emulated network interface.They are connected to the network, where the desktop delivery device ,the thin client,the fat client and the zero client reside via a network bridge module on the desktop delivery device.Like the desktop delivery device,thin client,fat client and zero client,each virtual desktop and virtual server has an IP address to communicate with the desktop delivery device,thin client,fat client and zero client. 


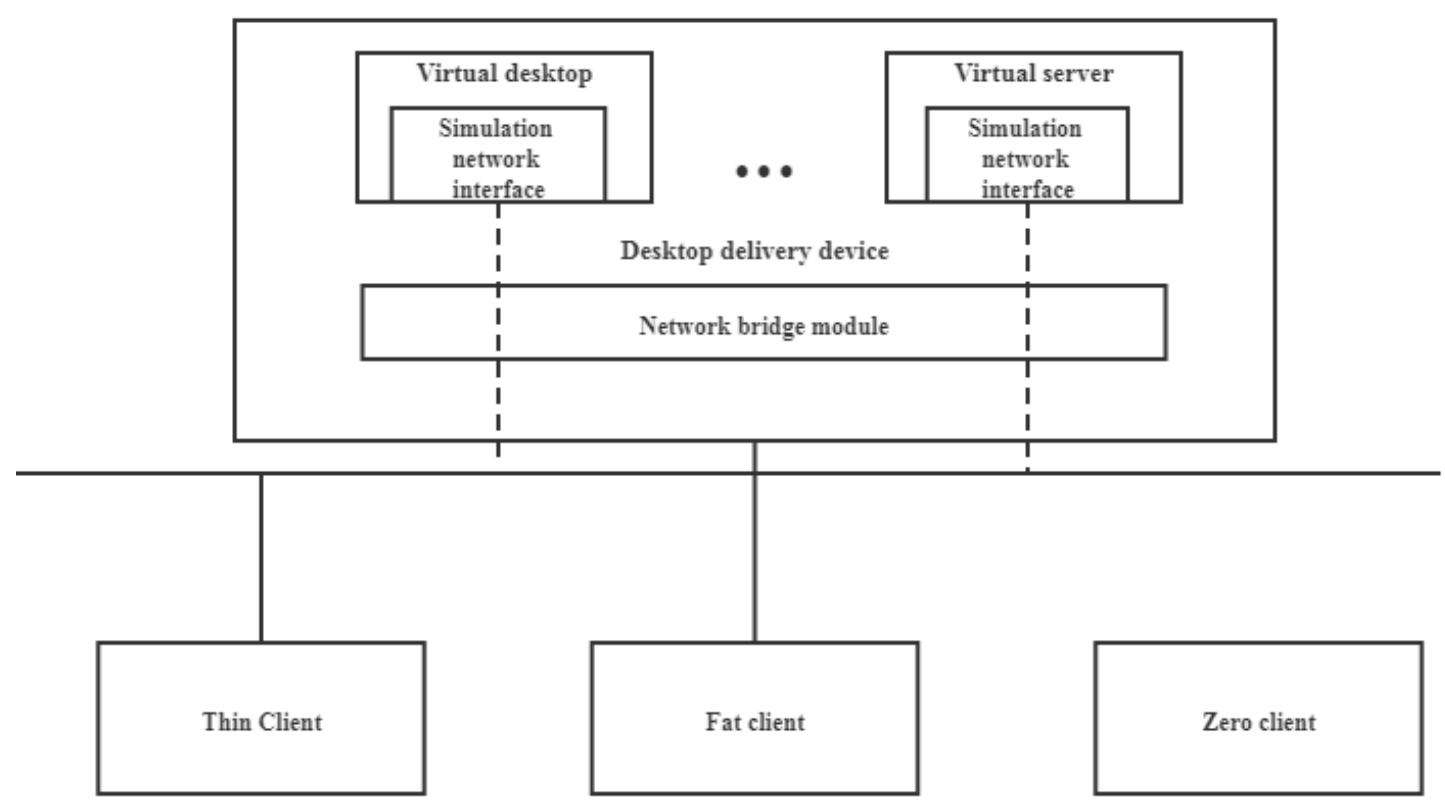

Fig. 3: Network Communication Diagram

Typically,thin clients install the client operating system and the connection protocol client. When the thin client is booted,the connection software will run automatically.The user logs in to the Desktop delivery device with an account and password,lists all the virtual desktops assigned to it and selects the virtual desktop to connect.Of course,thin clients can also automatically log in and connect to the virtual desktop.At the same time,thin clients can connect to virtual desktops which is running on desktop delivery devices via different protocols.

Fat clients install the client operating system.Due to the strong computing power,it can also run local virtualization software. When the fat client is booted,the desktop delivery device maps the desktop image to the fat client via a file mapping module (such as NFS or I SCSI), which is the desktop image mapped to the client.This allows the fat client to boot and run the desktop image mapped to the client in the local virtualization software.

The zero client runs locally by downloading the client image from a virtual server used for diskless boot technology.The zero client does not install the client operating system, unlike the thin and fat clients.The zero client uses the PXE protocol to boot from the network, with the help of a virtual server using diskless boot technology on the desktop device.Virtual server using diskless boot technology installs the virtual machine operating system, as well as the DHCP[9]module and the TFTP[10] module.The zero client obtains an available IP address through the DHCP module,and then downloads the corresponding client image through the TFTP module,that is to say,they are downloaded to the client image.In this way,the zero client can be booted.

\section{Conclusion}

The realization system of the desktop delivery device based on virtualization technology studied in this paper,by integrating VDI and VOI technologies and combining diskless boot technology,enables various clients to be supported under a virtual desktop architecture.The thin client connects to a virtual desktop running on a virtualized server via transport protocol connection software.The fat client gets the virtual desktop image from the virtualization server.Then this image runs in the local virtualization software.Through the network boot,the zero client gets the corresponding client image from the virtualization server.When the image runs, it can act as a thin client or fat client,using the virtual desktop on the virtualization server and building the desktop virtualization platform quickly and easily.Virtual desktop services can be provided for a variety of client devices simply by deploying the desktop delivery device on the LAN. 


\section{Acknowledgements}

Fund project: Cernet next generation Internet technology innovation Project NGII20170505;Funded by Shanghai Science and Technology Commission, project: Research on generic key technologies for smart family services, No14511108001

\section{Reference}

[1] L.Zhao. Implementation of Virtualization Technology of Android Emulator Operating System on PC. Science Technology and Engineering.2019,19(14):243-248.

[2] M.Salem, K.Samara, M.Aldhaheri. A novel integrated framework for securing online instructor-student communication. Int. J. of Grid and Utility Computing.2019,10(1):42-52.

[3] I.Khan,H. Rehman,M.Al-khatib,Z.Anwar,M.Alam. A thin client friendly trusted execution framework for infrastructure-as-a-service clouds. Future Generation Computer Systems.2018,89:239-248.

[4] Y.Zhang,S.Ling.Design and Practice of Multimedia Classroom Desktop Cloud integrated by VDI+VOI. Laboratory research and exploration.2017,36(10):145-148.

[5] J.Hu,W.Chen,L.Wu.Design and construction of composite cloud desktop for experimental teaching and students' innovative activities. Experimental technology and management, 2020,37(06):246-249.

[6] Y.Li,W.Jiang, J.Ji,D.Duan.Research on security outsourcing technology based on Virtualization Platform. Information network security.2018 (05): 82-88.

[7] R.Lin,W.Pan,S.Chen.Renovation of University Computer Laboratory of PXE Diskless Network Technology. Laboratory Research and Exploration.2018,37(01):241-246.

[8] R.Lin,Y.Lin,W.Pan,S.Chen.Application of Diskless Network Technology and Cloud Technology in Laboratory Construction.Experimental Technology and Management.2018,35(04):246-250.

[9] Z. Li. Backup and Migration of DHCP Server. Electronic World.2020(11):172-173.

[10] N.Mohamed,Y.Yussoff,M.Isa,H.Hashim. Extending hybrid approach to secure Trivial File Transfer Protocol in M2M communication: a comparative analysis. Springer US.2019,70(4):511-523. 